\title{
Rehabilitation of voice and speech after vocal prosthesis placement
}

\begin{abstract}
Loss of voice after total laryngectomy propter laryngeal cancer disables a person to successfully communicate with patient environment and can lead to total isolation. Learning esophageal speech in some cases is not successful. Vocal prosthesis insertion can resolve this condition. This retrospective study shows voice and speech rehabilitation in patients after total laryngectomy and installation of voice prostheses. The study included 48 patients ( 7 women, 41 men) with vocal prosthesis placement. Patients were 44-77years old (mean 62.5$)$. In 7 patients (14.58\%) underwent primary installation, and $41(85.42 \%)$ patients with secondary installation of a voice prosthesis. The results of comparative analysis of four basic parameters of voice was found that the maximum phonation time of tracheoesophageal speech $(12.5 \mathrm{~s})$ shorter than the normal speech $(23.5 \mathrm{~s})$, and longer compared with esophageal speech (3s). Height tracheoesophageal voice $(70-190 \mathrm{~Hz})$ is lower than the normal level of voice $(150-220 \mathrm{~Hz})$ and higher compared with the amount of esophageal voice $(70 \mathrm{~Hz})$. While the tracheoesophageal voice and speech needs more time for saying 120 phonetically balanced words $(2.20 \mathrm{~min})$ than normal voice and speech $(1.08 \mathrm{~min})$ and shorter than esophageal speech $(3.05 \mathrm{~min})$.
\end{abstract}

Conclusion: Tracheoesophageal voice and speech with voice prosthesis placement have extremely high success rate (98\%), faster rehabilitation and better functional results than esophageal speech.

Keywords: total laryngectomy, voice prosthesis, voice rehabilitation
Volume 2 Issue 4 - 2015

\author{
Tanja Arbutina,' Rajko Jovic, ${ }^{2}$ Jugoslav Gasic, ${ }^{3}$ \\ Danijela Dragicevic ${ }^{2}$ \\ 'Phoniatric department of ENT Clinic, Clinical CenterVojvodina, \\ Serbia \\ ${ }^{2}$ University ENT Clinic, Novi Sad, Serbia \\ ${ }^{3}$ ENT Clinic, Medical school K. Mitrovica, Serbia
}

Correspondence: Tanja Arbutina, Speech and Language pathologist, Hajduk Veljkova 2, ORL Klinika, Novi Sad, Serbia,Tel +381 64 2309064, Email atanjnja@gmail.com

Received: March 19,2015 | Published: April 25, 2015

\section{Introduction}

The highest incidence of malignant tumors of the head and neck are tumors of larynx. ${ }^{1}$ They require surgical-medical treatment in the form of total laryngectomy with or without additional surgical resection. After a surgical intervention such as a total laryngectomy, permanent damage of phonetic automatism takes place, a patient faces swallowing problems, loss of a nasal function while the lungs function changes. ${ }^{2}$ This refers to a period during which a patient is voiceless and without an ability to speak. Such a condition is permanent thus requiring an immediate rehabilitation. One type of the voice and speech rehabilitation is placement of a voice prosthesis, which may enable a patient a verbal communication within a period of few days. The voice prosthesis is a one-way valve, which enables a free air friction in esophagus and pharynx. This enables a light production of functional traheoesophageal voice and speech. Placement of the voice prosthesis may be primary or secondary. This depends on the economic situation of the society, pre-intervention assessment of a patient's speech abilities and the advancement level of phonation function. Primary installation is carried out during the surgical intervention by removing the affected larynx, while a secondary installation is carried out after the phonetic rehabilitation of laryngectomized patient had failed. The assessment of the speech abilities is carried out by applying the pre-intervention procedure. The speech rehabilitation within the primary installation of a voice prosthesis starts around tenth day after the intervention, i.e. once the operation wound has healed, while the secondary rehabilitation can start the very next day. The objective is to show how to carry out the post-surgical speech rehabilitation, how to achieve the quality voice and speech as well as to present the issues faced during the rehabilitation procedure.

\section{Materials and methods}

The average age of 48 patients (7women and $41 \mathrm{men}$ ) with a voice prosthesis installed is 62.5 (44-77years). Seven primary and 41 secondary prostheses were installed. The primary installation was applied to younger patients that have an ability to work and that will, thanks to the good speech quality, be able to continue with their regular work and life activities. The program of secondary installation included all patients whose training of esophageal voice and speech after six weeks was evaluated as unsuccessful. Those patients had not managed to adopt the main elements of esophageal voice and speech or they had not managed to automate it thus resulting in a lack of establishment of verbal communication. For those patients, the installation of the voice prosthesis enables re-establishing of the verbal communication which characteristics are fully aligned with the regular ones. Objective acoustic analysis of each patient voice was done. For these purposes, we used the software Voice assessment, Dr Speech. The patient is sitting in a room isolated from the noise, and on the microphone, which has been located by the mouth 5 centimeters, in a given moment, said vowel-a-. In this way, the amount determined in patients votes. Maximum phonation time represents an aerodynamic during eructation. Maximum phonation time represents the aerodynamic test phonation. It represents the maximum time possible, in this case saying vocals. After patient placed air into the esophagus he said vocal-a-, and time is measured in seconds. Assessment of speech was assessed by measuring the length of the reading phonetically balanced text. The patient was reading the text "Hello Mark", a reading time is measured by the clock with seconds. Spontaneous speech is evaluated based on the number of words in one eructation. Patient was told what he was doing during the day, while the examiner recorded the number of understandable words spoken during an eructation.

The voice and speech training of the patients with the primary installation of the speech prosthesis usually starts on the tenth day after the surgery, i.e. when the healing process is completed without any complications. The secondary installation was carried out 
upon the completion of different oncological treatments. A patient was registered at the department of the Clinics. During the total endotracheal anesthesia, a small surgical intervention is carried out, which establishes the communication between tracheostoma shunt and esophagus where the vocal prosthesis is placed. The patients were directed to the speech treatment the day after the intervention in case the immediate post-intervention process was regular.

\section{Rehabilitation procedure}

Preparation of a patient for the beginning of the speech rehabilitation: In the beginning is important for a patient to receive sufficient information about all characteristics of the speech prosthesis and it capabilities prior to its placement. It is particularly important to highlight to a patient that the spontaneous speech does not start immediately upon the placement of the prosthesis but one has to learn to speak and the learning procedure is significantly shorter. In that phase, support from the immediate family is very important as to decrease and overcome the side effects that could appear during the treatment. It is necessary to create a positive atmosphere in the patient's microenvironment that will contribute to the successful rehabilitation treatment.

\section{Speech rehabilitation}

Day one: In-the hospital, the speech treatment was conducted twice a day. During the first day, the sessions are shorter and they last around ten minutes. The objective of the exercise during the first day is for a patient to sound exhaling air and to learn how to coordinate fingers by pressing a button of a filter and exhale. After informative discussion with the patient about the possibilities and functions of the voice prosthesis, hygiene and its maintenance, the treatment may start. The patient sits in a relaxed position against a mirror to be able to follow the coordination of his hand, breathing and speech. The first exercise begins with the pronunciation of a vocal -a-. The patient is being taught to press a button on a filter at the moment of exhaling and to exhale the air in the direction of the prosthesis. The air becomes sounded by passing through the prosthesis hole in the upper part of esophagus and pharynx, while in an oral cavity the vocal -a- is formed. Other vocals as well as the combination of the vocals with $-\mathrm{h}$ - are pronounced in the same manner.

Day two: The rehabilitation continues with pronunciation of selected words containing short vocals followed by the selected words with longer vocals.

Day three: A patient is asked to pronounce selected simple sentences in a form of questions followed by selected complex sentences. The patient counts from one two five within one breathe.

Day four: A patient has learned how to pronounce complex sentences containing four to five words, therefore the rehabilitation continues with reading a phonetically balanced text. The period of four days is usually also a period necessary for the speech rehabilitation for patients with voice prosthesis. Very rarely the rehabilitation required additional engagement. During the last rehabilitation day, a patient reads simple texts, interprets them and slowly automates newly learnt voice and speech into spontaneous verbal communication.

\section{Results}

All patients had their larynx removed in total, while some of them had undergone additional surgical resection of neighboring cancer- affected structures (Table 1). Both placement of the voice prosthesis and rehabilitation procedure were carried out during the period of 5 days. At the day of installation, the rehabilitation of voice and speech was not conducted - it was first applied only after the first postoperation day. The secondary installation of the voice prosthesis was carried out from 3-98 months after a cancer operation (in average 20.8 months). When it comes to patients with advanced cancer, 33 (68.7\%) of them had an indication for post operation air therapy that was carried out eventually. Irradiated tissue changes its characteristic, thus the edema and the neck rigidity are regular and influencing the course of rehabilitation. The period upon the completion of the air therapy until the installation was 15.6 months (2-96 months) in average.

Table I Scale of resection of the structures that participate in forming of a voice and speech

\begin{tabular}{llc}
\hline Type and Scale of Resection & N & $\%$ \\
\hline $\begin{array}{l}\text { Complete Resection Of Larynx } \\
\begin{array}{l}\text { Complete Resection Of Larynx With Partial } \\
\text { Resection Of Pharynx }\end{array}\end{array}$ & 40 & 83.3 \\
$\begin{array}{l}\text { Complete Resection Of With Resection Of The } \\
\text { Basis Of A Tongue }\end{array}$ & I & 12.5 \\
$\begin{array}{l}\text { Complete Resection Of Larynx, Lower Part Of } \\
\text { Pharynx And Esophagus (Reconstruction Of } \\
\text { Esophagus With Gaster) }\end{array}$ & I & 2.1 \\
\begin{tabular}{l} 
Total \\
\hline
\end{tabular} & 48 & 100 \\
\hline
\end{tabular}

Not even one complication was registered during the surgical intervention of installing the voice prosthesis. During the rehabilitation period (Table 2), the most frequent complication was hypertonicitiy of cricopharyngeal muscle at 7 (14.6\%) patients. All patients had cricopharyngeal muscle infiltration with local anesthetic Lidocaine $2 \%$. Five patients, after two days of the anesthetics, had their voice established and with the further rehabilitation procedure came to spontaneous speech, while two patients had undertaken additional radiologic research, after which the surgical resection of cricopharyngeal muscle was indicated. During the total endotracheal anesthesia, a vertical resection of cricopharyngeal muscle was carried out. One patient had a positive effect of the resection and he was provided with phonation and quality speech. With the other patient any post-intervention rehabilitation treatment or any repeated later attempts with neck infiltration with local anesthetics did not result in establishing of phonation or articulation. After five days of hospitalization and ten speech treatments, the patients were trained for verbal communication with tracheoesophageal voice and speech and released from the Clinics. In case of the difficulties during rehabilitation, it was performed $\mathrm{X}$-ray diagnostics, and in therapy was performed infiltration with Xylocaine in cricopharingeal muscle.

The speech treatment continues upon the release from the hospital, once a week during a one-month period. The treatments included exercises to increase the quality of the speech characteristics. The assessment of the acquired speech was carried out by applying four standard parameters. The values were compared with the ones acquired from esophageal and normal speech (Table 3).

In $47(97.9 \%)$ patients the rehabilitation procedure resulted in the aimed verbal communication. The quality of articulation, acoustic impression and the level of understanding of the speech were satisfactory. 
Table 2 Influence of radiotherapy on rehabilitation efficancy

\begin{tabular}{lll}
\hline & Pree-Irradiation & No Previous Radiotherapy \\
\hline Rehabilitated Patient & $32(66.6 \%)$ & $15(31.25 \%)$ \\
Failed with Rehabilitation & $\mathrm{I}(2.08 \%)$ & 0 \\
\hline
\end{tabular}

Table 3 Comparative values of voice and speech per different categories

\begin{tabular}{llll}
\hline & $\begin{array}{l}\text { Traheooeshophageal } \\
\text { Speech Prothesis }\end{array}$ & $\begin{array}{l}\text { Oeshophageal } \\
\text { Speech }\end{array}$ & Normal Speech \\
\hline $\begin{array}{l}\text { Maximal Phonation Time (Seconds) } \\
\begin{array}{l}\text { Time Necessary To Pronounce I20 } \\
\text { Phonetically Balanced Words (Minutes) }\end{array}\end{array}$ & $4-21$ & $2-4$ & $12-35$ \\
$\begin{array}{l}\text { Number OfWords During Exhalation } \\
\text { Voice Frequency }(\mathrm{Hz})\end{array}$ & $8-10$ & $1.5-5.5$ & $1.06-1.1$ \\
\hline
\end{tabular}

\section{Discussion}

Loss of voice disables a person to successfully communicate with patient environment. Sometimes it leads the person to total isolation. Larynx communication is motoric action that needs to be learnt. As phonation, it is developed, controlled by acoustic, auditive and kinestatic feed-back system and it is being driven by central system. The voice condition after total laryngectomy leads to disturbed adoptive behavior. As a consequence of the voice deficit, a patient experiences difficulties in adopting to the requirements of the environment, in developing interpersonal relations, communication capabilities, as well as in adjusting to social and work environment. This mostly refers to taking care of his needs. We noticed that patient with secondary placement of voice prostheses are more motivated then patients with had primary placement of voice prostheses due to they had not have period of apfonia. The beginning of the rehabilitation is easier and faster with the placement of the voice prosthesis. The exercises started immediately, provoking vocals. To develop the skills of esophageal voice and speech, first it is necessary to choose one of the four methods that best suit a patient to obtain spontaneous burps. It usually begins with a deglutition method, which was later diverted to aspiration method, because the act of swallowing with aging weakens, thus esophageal voice and speech decline. It is important to control contraction of muscles during speech. They have to economize air max, which indicates the importance of preservation of executive function and implicit memory. Forced phonation is one of the reasons that the rehabilitation of esophageal voice and speech declared as failed, and it can be seen in the same patient who later joined with placement of the voice prosthesis. It is solved by local infiltration of $2 \%$ lidocaine in the neck muscles. When speaking with voice prosthesis phonation automatisms are not decomposed because the lungs are still the activator of voice. Therefore, the verbal rehabilitation of such patients is easier. Esophageal voice is the best solution for a patient who can learn it. It does not require additional surgery, requires no equipment and during its use, patient hand activity is not required. ${ }^{3}$ The lack of it is a very long rehabilitation that lasts approximately 6 months. After total laryngectomy patients do not have normal lung volumes less than $3000 \mathrm{ml}$ of phonation due to breathing trough the tracheostomy. Reservoir formed by the patient's speech in the area of the lower part of the pharynx and upper esophagus with $80 \mathrm{ml}$ of air. ${ }^{4}$ It is a small amount of air that does not allow long phonation and articulation. It is an important comparative advantage of voice prosthesis can use entire lung volume. The placement of voice prosthesis mass was started in the world 1979 when it appeared Blom-Singer prosthesis. ${ }^{5}$ It has its advantages and disadvantages that may impact the course and outcome of rehabilitation process. The main disadvantages are the need for additional surgery and the high cost of prosthesis. The patient must use finger and have good coordination with hands while using it. The advantage is short rehabilitation, fluent and exceptionally high success rate, in our patients even 47/48 (98\%). Successful rehabilitation is realistic to expect in patient esophageal voice and speech, if they start rehabilitation in period from 2 to 4 months after surgery. In patients who have applied for rehabilitation after year or more, the success of rehabilitation are not realistic due to the change patterns of phonation automatisms, automated whisper. This is not relevant to the traheoesophageal voice and speech after placement of voice prosthesis. It differs from the ratio of successful rehabilitation and the necessary number of exercises in these two ways of speaking. With vocal prosthesis should be between 10 and 20 vocal exercises in order to establish a pragmatic and functional voice and speech and over 30 exercises in order to improve quality of voice and speech. Esophageal voice and speech variation, in intensity and tonality after completing rehabilitation are minimal. Acoustically, it looks more like noise than sound and shows significant deficiencies in relation to normal and trahoesophageal voice and speech using voice prosthesis, where it achieved considerable variation in the amount and intensity.

Steffen found the amount of tracheoesophageal voice prosthesis with a vocal range of $80-110 \mathrm{~Hz}$ which is almost the same as our results. In further states in his study he report that in patient with vocal prosthesis phonation time (Table 4) is 10.05 seconds, which is much longer than the phonation time of patient with esophageal voice and speech, where is 1.6 seconds. ${ }^{5}$ Also Bozec et al., ${ }^{2}$ states that the maximum phonation time in patients with vocal prosthesis ranges from 12-13seconds, and in our study maximum phonation time is longer and amounts $12-21 \mathrm{~s}$. In the world literature states that patients with vocal prosthesis can say up to 150 words a minute, a number of words in one breath is 16words, and in esophageal speech is 5 words $s^{5-7}$ which is the same as in our results for esophageal speech. 
Table 4 Average maximal phonation time of the vocal -a- per days

\begin{tabular}{llll}
\hline & First Day & Third Day & Fourth Day \\
\hline $\begin{array}{l}\text { Average Maximal Phonation Time Of The } \\
\text { Vocal A (Seconds) }\end{array}$ & $2 \mathrm{sec}$ & $5 \mathrm{sec}$ & $12 \mathrm{sec}$ \\
\hline
\end{tabular}

So far unsuccessful rehabilitation of esophageal speech we considered when a patient after three weeks of hospital treatment (30 exercises) has not mastered the voice and speech. In the meantime, we have introduced prediction parameters for successful rehabilitation of esophageal voice and speech, namely: shape of hipopharingeal funnel, the absence of hypertonicity (light insufflation), extent of resection of the mucous membrane of the pharynx, (laryngectomy or pharyngectomy). On this basis, we have reduced learning esophageal voice and speech for three weeks, and made indication for the installation of secondary placement of voice prostheses. Hypertonicity is present in a small number of patients in the education of esophageal voice and speech, and in these cases we manage infiltration with Lidocaine. In these patients more detailed results from these treatments are yet to come.

\section{Conclusion}

The voice and speech rehabilitation after placing the voice prosthesis requires a team approach. For the success of the rehabilitation it is necessary to systematically implement speech and language treatment trough the certain of affirmative atmosphere. Surgical placement of the voice prosthesis is the best alternative for developing the larynx communication. The rehabilitation conducted in a hospital and ambulatory treatments lasting one month. The voice prosthesis affects quality life improvement for a laryngectomized patient.

\section{Acknowledgments}

None.

\section{Conflicts of interest}

The authors declare that there are no conflicts of interest.

\section{Funding details}

None.

\section{References}

1. Casper C, Schuster M, Psyhogios G, et al. Voice hendicep index and voice-releted quality of life in small laryngeal carcinoma. Eur Arch Otorhinolaryngol. 2011;268(3):401-404.

2. Bozec A, Poosonet G, Chamoery E, et al. Results of vocal rehabilitation using tracheoeosophageal voice prothesis after total laryngectomy and their predictive factors. Eur Arch Otorhinolaryngol. 2010;267(5):751-758.

3. Pandzola RH, Cain BH. Duration and frequency characteristic of tracheoesophageal speech. Ann Otol Rhinol Laryngol. 1989;98(12 Pt 1):960-964.

4. Kramp B, Dommerich S. Tracheostomy cannulas and vioce prosthesis. GMS Current topic in Laryngorhinootologie. 2009;(88Suppl1):S95S118.

5. Robins J, Fisher HB, Blom EC, et al. A comparative acostic study of normal, esophageal and tracheoesophageal speech production. $J$ Speech Hear Disord. 1984;49(2):202-210.

6. Max L, Steurs W, de Bruyn W. Vocal capacities in esophageal and tracheoesophageal speakers. Laryngoscope. 1996;106(1 Pt 1): 93-96.

7. Gerwin JM, Culton GL. Quality of life in prosthetic voice users. Otolaringol Head Neck Surg. 2005;133(5):685-688. 